\title{
Tamaño óptimo de muestra del programa de observadores a bordo de la flota dirigida a la explotación de la anchoveta peruana (Engraulis ringens)
}

\section{Optimum sample size for a program of observers on board fishing vessels targetting Peruvian anchovy (Engraulis ringens)}

\section{Rocío Joo ${ }^{1 *}$, Daniel Grados ${ }^{1,2}$, Marilú Bouchon ${ }^{1}$ y Erich Díaz ${ }^{1}$}

1 IMARPE, Instituto del Mar del Perú, Esquina Gamarra y General Valle S/N, Chucuito, Callao, Perú.

2 Institut de Recherche pour le Développement (IRD), Centre de Recherche Halieutique Méditerranéenne et Tropicale, Avenue Jean Monnet, BP 171, 34203 Sète Cedex, France.

* Autor para correspondencia

E-mail Rocío Joo: rocio.joo@gmail.com

E-mail Daniel Grados: danny.grados@gmail.com

E-mail Marilú Bouchon: mbouchon@imarpe.gob.pe

E-mail Erich Díaz: ediaz@imarpe.gob.pe

\begin{abstract}
Resumen
El monitoreo de la pesquería de anchoveta en el Perú es de suma importancia para la sostenibilidad del ecosistema de Humboldt. El Programa de observadores a bordo Bitácoras de Pesca constituye una rica plataforma de recolección de datos de las embarcaciones durante sus viajes de pesca, y donde se recopila información acerca de las capturas en cada lance, distribuciones de tallas, descartes, captura incidental, captura por unidad de esfuerzo, entre otros. Para que los indicadores obtenidos a partir de esta información tengan robustez es preciso contar con un método de solidez estadística para el cálculo del número necesario de viajes a muestrear. Este trabajo presenta un método con estas características, para calcular el número óptimo de viajes de manera independiente para cada una de las variables y a diferentes escalas de tiempo. Se muestra que, dependiendo del objetivo y de la escala temporal, la cantidad óptima de viajes a muestrear varía. A partir de estos resultados, se proporcionan recomendaciones prácticas sobre el tamaño de muestra para el Programa Bitácoras de Pesca. También se discute la aplicabilidad de esta metodología para otros recursos pelágicos.
\end{abstract}

Palabras claves: tamaño de muestra; simulaciones; observadores a bordo; pesquería pelágica de cerco; anchoveta; Engraulis ringens.

Citación:

Joo R., D. Grados, M. Bouchon y E. Díaz. 2016. Tamaño óptimo de muestra del programa de observadores a bordo de la flota dirigida a la explotación de la anchoveta peruana (Engraulis ringens). Revista peruana de biología 23(2): 169 - 182 (Agosto 2016). doi: http://dx.doi. org/10.15381/rpb.v23i2.12429

$\begin{array}{ll}\text { Presentado: } & 31 / 12 / 2015 \\ \text { Aceptado: } & 01 / 07 / 2016\end{array}$

Publicado online: $27 / 08 / 2016$
Información sobre los autores:

RJ: contribuyó en la concepción, redacción del manuscrito, participó en el diseño de la metodología, realizó algoritmos y cálculos para el trabajo. DG: participó en el diseño de la metodología, en los algoritmos y cálculos para el trabajo. MB: participó en la redacción del manuscrito. ED: contribuyó en la concepción, facilitó los datos históricos, participó en la redacción del manuscrito. RJ, DG, MB y ED aprobaron el manuscrito. Los autores no incurren en conflictos de intereses.

Fuentes de financiamiento: (1) Proyecto PpR "Observación y evaluación en tiempo real del subsistema pelágico del Ecosistema de la Corriente de Humboldt utilizando como plataforma la flota de cerco" del IMARPE - financiado con recursos de Derechos de Pesca (RM-N ${ }^{\circ}$ 235-2013-PRODUCE), (2) Proyecto PpR “Estimación de parámetros biológico-pesqueros para el manejo sostenible de los recursos marinos"del IMARPE- financiado con recursos de Derechos de Pesca (RM-350-2013-PRODUCE) 


\begin{abstract}
Monitoring the anchovy fishery is of great importance for assuring the sustainability of the Humboldt ecosystem. The on-board observers program constitutes a rich platform for data collection for monitoring, as it consists of the collection of data from vessels during their fishing trips, regarding catches, size distribution, discards, catch per unit of effort, among others. To get robust indicators from these data, it is necessary to use a solid statistical procedure for computing the number of fishing trips to sample. This work presents a method with those characteristics, for computing each indicator independently and at different time scales. We show that the optimum size varies depending on the objective (indicator) and the time scale. Based on these results, practical recommendations for fixing the sample size are given. We finally discuss the aplicability of this methodology for other pelagic resources.
\end{abstract}

Keywords: sample size; simulations; on-board observers; purse-seine pelagic fishery; anchovy Engraulis ringens.

\section{Introducción}

La pesquería de recursos pelágicos como la anchoveta (Engraulis ringens) (principalmente destinada al consumo humano indirecto), el jurel (Trachurus murphyi) y la caballa (Scomber japonicus) (generalmente destinados al consumo humano directo) tiene repercusiones económicas, ecológicas y sociales muy importantes para el Perú (Aranda 2009, Tveteras et al. 2011, Avadí et al. 2014). Entre estas especies, la anchoveta es la principal especie capturada, pues representa $\sim 95 \%$ de las capturas (Fréon et al. 2008). Para garantizar la sostenibilidad de este y otros recursos son claves el monitoreo del recurso, de la actividad extractiva sobre él y del ecosistema. Actualmente, el monitoreo de los recursos pelágicos es intensivo, lo que permite el manejo adaptativo de las pesquerías (Chavez et al. 2008, Arias Schreiber et al. 2011). El sistema de monitoreo comprende diversas plataformas de recolección de datos, entre las cuales se destacan las observaciones a bordo de las embarcaciones que conforman la flota de cerco. Estas observaciones se realizan en el marco de un programa de observadores a bordo denominado Programa Bitácoras de Pesca (PBP) que el Instituto del Mar del Perú (IMARPE) viene realizando desde 1996. La información obtenida por este programa permite, entre otras cosas, caracterizar la dinámica de la flota, cuantificar los descartes, caracterizar la captura incidental, describir el comportamiento de los recursos, su distribución y estructura demográfica y cuantificar la interacción con los predadores superiores, entre otros.

Los datos recolectados por este programa enriquecen la evaluación y gestión del recurso. Sin embargo, el alto costo de enviar observadores a bordo de las embarcaciones a menudo implica que solo un pequeño número de viajes pueden ser observados (Cotter et al. 2002). Históricamente, el número de viajes muestreados en el PBP ha obedecido a restricciones presupuestarias. No obstante, es necesario determinar el tamańo de muestra mínimo que garantice una estimación insesgada y precisa de los indicadores que se calculan a partir de los datos recolectados.

Existen muy pocos trabajos que evalúan la cobertura de los observadores a bordo para estimar descartes, captura incidental, estructura de tallas, entre otros. Entre ellos, lo más usual es utilizar modelos jerárquicos multinivel que consideren los efectos a nivel de barco, lance, y/o ejemplar (e.g. Smith \& Baird 2005,
Montenegro Silva 2009). Para elegir un tamaño de muestra óptimo, se han utilizado distintos criterios. Smith y Baird (2005) utilizaron un modelo jerárquico de captura incidental para calcular un coeficiente de variación y fijaron como óptimos aquellos tamańos de muestra mínimos con los que se obtuvieron coeficientes de variación del 10, 20 y 30\%. Montenegro Silva (2009), en cambio, modeló tallas de captura (del langostino colorado Pleuroncodes monodon) y comparó las frecuencias relativas de las tallas estimadas con las observadas utilizando un índice de error (Andrew \& Chen 1997) similar a una suma cuadrática media; también se calculó el error en la estimación de la talla media. El tamaño óptimo de muestra fue aquel a partir del cual los indicadores convergieron. Considerando múltiples objetivos de estimación a la vez (captura incidental, captura total y estructura de tallas), Miller et al. (2007) calcularon el tamańo de muestra en función a las varianzas relativas de las estimaciones, que fueron ponderadas de acuerdo a la importancia de cada objetivo; también se consideraron para el cálculo los costos del muestreo para cada objetivo y en cada etapa del muestreo. Por otro lado, en lugar de ajustar modelos utilizando datos históricos, Babcock y Pikitch (2003) simularon pesquerías con captura incidental de diversas especies y determinaron porcentajes de cobertura distintos para los viajes de pesca de especies raras respecto a aquellos de especies comunes, para garantizar altos niveles de precisión en las estimaciones de captura incidental.

En este trabajo se calcula el número óptimo de viajes que deberían de realizar los observadores a bordo del Programa Bitácora de Pesca, para responder a diferentes objetivos de evaluación de la pesquería de cerco pelágica de anchoveta. Los datos recolectados por el programa responden a múltiples objetivos, por lo que interesa obtener estimaciones precisas e insesgadas de múltiples variables. Dado que cada objetivo varia de un año a otro, se calculará un tamaño óptimo de muestra para cada objetivo independientemente de los otros. Como en Babcock y Pikitch (2003), se utilizará un enfoque de simulación estocástica. Los datos históricos del PBP serán utilizados para calcular los parámetros de las distribuciones con las que se realizarán simulaciones de las variables. A continuación, presentamos una breve descripción del PBP y de las variables e indicadores que se calcularán a partir de las observaciones del PBP. Luego, 
describiremos la metodología y determinaremos el número de viajes necesarios para cada uno de los indicadores. Finalmente, discutiremos la aplicabilidad de esta metodología para otros recursos pelágicos monitoreados por el $\mathrm{PBP}$ y proporcionamos algunas recomendaciones para mejorar la representatividad de la información recabada por el programa.

\section{Material y métodos}

Programa Bitácoras de Pesca.- El PBP es una plataforma de monitoreo de la pesca pelágica de cerco en el Perú. La mayor parte de las observaciones realizadas corresponden a viajes de las embarcaciones industriales dedicadas a la pesca de anchoveta. El número de viajes de embarcaciones industriales dedicadas a la pesca de anchoveta que fueron muestreados anualmente ha variado entre 106 (en el 2010) y 1467 (en 1997) (Tabla 1). Durante cada temporada de pesca, cada observador debe instalarse a bordo de una embarcación de la flota de cerco y permanecer en ella durante el número de días de trabajo asignado por mes ( -20 días). Como no es obligatorio que las embarcaciones acepten observadores a bordo, la coordinación para el embarque se realiza entre el IMARPE y la empresa dueña de la embarcación de manera voluntaria, días antes de iniciar la temporada de pesca.

Durante cada viaje, el observador anota en una ficha denominada "Bitácora de Pesca" datos sobre el viaje, los lances y la embarcación. Desde 1996 hasta el 2007, los observadores registraron sobre cada viaje principalmente: puerto y tiempo de salida y arribo, el tiempo de las diferentes actividades realizadas durante los viajes, la captura total, los depredadores superiores observados y las características biométricas de las especies capturadas. Después del 2007, también fueron registradas: la compañía pesquera, características de la infraestructura y equipos de las embarcaciones, del capitán, captura descartada, ofrecida, recibida, criterios empleados para escoger las zonas de pesca, detección de predadores durante el viaje, entre otros.

Los registros se realizan tanto para la pesca en la región NorteCentro (desde $43^{\circ} \mathrm{S}$ hasta $16^{\circ} \mathrm{S}$ ) como el en Sur (desde $16^{\circ} \mathrm{S}$ ). El manejo de la pesquería en cada región se realiza de manera independiente. Como el $90 \%$ de la pesca se realiza en la región NorteCentro, para este trabajo sólo nos enfocaremos en esta región.

Objetivos e indicadores.- Los indicadores que se calculan a partir del PBP y que contribuyen al seguimiento de la pesquería corresponden a diferentes escalas temporales (diarias, mensuales, anuales) y responden a diferentes objetivos de estimación. Las escalas corresponden a los periodos en los que esos indicadores son utilizados para el monitoreo. Los objetivos de estimación se pueden clasificar en:

1) Talla de anchoveta capturada (escala mensual). Los indicadores calculados a partir de esta variable fueron el promedio y la desviación estándar. A partir de esa información se monitorea la proporción del stock que está siendo removida por la pesca y se investigan los cambios en la estructura demográfica del stock. Esta información también es utilizada en la evaluación de la anchoveta.

2) Ocurrencia de juveniles en las capturas de anchoveta (escala diaria). El indicador calculado fue el porcentaje de ocurrencia de juveniles. A partir de esa información se monitorea la mortalidad por pesca de los juveniles y se identifican las áreas con alta ocurrencia de juveniles para recomendar la suspensión de la pesca en ellas.
Tabla 1. Tamaños de muestra anuales tomados entre 1996 y 2014 para la pesquería industrial de anchoveta para las regiones Norte-Centro y Sur, y el total.

\begin{tabular}{cccc} 
& \multicolumn{2}{c}{ Región } & \multirow{2}{*}{ Total } \\
\cline { 2 - 3 } $\mathbf{1 9 9 6}$ & Norte-Centro & Sur & \\
$\mathbf{1 9 9 7}$ & 906 & 53 & 959 \\
$\mathbf{1 9 9 8}$ & 1273 & 194 & 1467 \\
$\mathbf{1 9 9 9}$ & 735 & 45 & 780 \\
$\mathbf{2 0 0 0}$ & 803 & 139 & 942 \\
$\mathbf{2 0 0 1}$ & 631 & 56 & 687 \\
$\mathbf{2 0 0 2}$ & 707 & 78 & 785 \\
$\mathbf{2 0 0 3}$ & 1181 & 233 & 1414 \\
$\mathbf{2 0 0 4}$ & 482 & 77 & 559 \\
$\mathbf{2 0 0 5}$ & 643 & 208 & 851 \\
$\mathbf{2 0 0 6}$ & 1069 & 261 & 1330 \\
$\mathbf{2 0 0 7}$ & 732 & 155 & 887 \\
$\mathbf{2 0 0 8}$ & 424 & 160 & 584 \\
$\mathbf{2 0 0 9}$ & 715 & 136 & 851 \\
$\mathbf{2 0 1 0}$ & 451 & 35 & 486 \\
$\mathbf{2 0 1 1}$ & 79 & 27 & 106 \\
$\mathbf{2 0 1 2}$ & 376 & 43 & 419 \\
$\mathbf{2 0 1 3}$ & 250 & 54 & 304 \\
$\mathbf{2 0 1 4}$ & 1026 & 27 & 1053 \\
\hline
\end{tabular}

3) Descartes de anchoveta por exceso de captura (escala anual). Los indicadores calculados fueron el porcentaje de viajes con descartes de anchoveta por exceso de captura, el promedio y la desviación estándar del volumen descartado. El descarte por exceso de captura es uno de los componentes de la captura no registrada en la pesquería y sólo se puede estimar a partir de la muestra de datos de observadores a bordo o a partir de las declaraciones de los pescadores en el momento del desembarque.

4) Descartes de juveniles de anchoveta (escala anual). Los indicadores calculados fueron el porcentaje de viajes con descartes de juveniles de anchoveta, el promedio y la desviación estándar del volumen descartado. El descarte de juveniles es otro de los componentes de la captura no registrada en la pesquería. Igualmente, sólo se puede estimar a partir de la muestra de datos de observadores a bordo o a partir de las declaraciones de los pescadores en el momento del desembarque.

5) Captura incidental de otros recursos (escala anual). Los indicadores calculados fueron el porcentaje de viajes con captura incidental, el promedio y la desviación estándar del volumen de captura incidental. La captura incidental es otro componente de la captura no registrada en la pesquería.

6) Profundidad de los cardúmenes de anchoveta (escala mensual y anual). Se calcularon como indicadores el promedio y la desviación estándar de la profundidad. Estos datos dan información sobre el comportamiento vertical de la anchoveta capturada (es decir, disponible y atractiva para los pescadores), lo que podría estar sujeto a cambios en las condiciones ambientales.

7) Duración de los viajes de pesca (escala mensual y anual). Los indicadores calculados fueron el promedio y la desviación estándar de la duración. A partir de esa información se describe el comportamiento de las flotas dirigidas a la explotación de la anchoveta y se cuantifica el esfuerzo pesquero desplegado. 
8) Captura por unidad de esfuerzo (CPUE) de las embarcaciones (escala mensual y anual). La CPUE fue calculada como la captura dividida por la duración del viaje. Los indicadores calculados fueron el promedio y la desviación estándar de la CPUE. A partir de esta información se complementa la descripción del comportamiento de la flota y se estima la abundancia relativa del Stock Norte-Centro de la anchoveta.

La Tabla 2 resume los indicadores que se calcularon y para los cuales se analizó la sensibilidad de la estimación al tamaño de muestra.

Metodología estadística.- Como la unidad de muestreo es el viaje observado, se analizó la sensibilidad de las estimaciones de cada uno de los indicadores al número de viajes muestreados por el PBP. De manera general, el procedimiento fue el siguiente:

1) Se realizaron simulaciones aleatorias de los valores poblacionales de los indicadores (es decir, los valores calculados a partir del total de viajes de pesca realizados por la flota). Los indicadores poblacionales fueron simulados utilizando parámetros obtenidos de los datos históricos del PBP y están descritos en el Anexo 1.

2) Se muestrearon $n$ viajes - simulando de esta manera el muestreo del PBP.

3) Usando los n viajes del muestreo se calcularon los indicadores descritos en la Tabla 2.

Los pasos 2 y 3 se realizaron $k$ veces $(k=1000)$. Utilizando los $\mathrm{k}$ resultados de los indicadores se construyeron los intervalos de confianza de las estimaciones y se calculó el error cuadrático medio (ECM) para evaluar el sesgo y la precisión para cada indicador. Cada indicador fue calculado para una escala temporal distinta, como se precisa en la sección de definición de descripción de objetivos e indicadores. Los detalles de las simulaciones y estimaciones se presentan a continuación:

\section{Estimación del promedio y la varianza de la estruc- tura de tallas de la capturas de anchoveta}

Simulación de la población y cálculo de los parámetros poblacionales.- Para una población de $\mathrm{N}$ viajes de pesca en el mes, primero se simuló el número de lances en cada viaje. Para cada lance, se simuló la estructura de tallas. Luego, se calculó la media y desviación estándar poblacionales de las tallas.

Muestreo para cada tamaño de muestra $\boldsymbol{n}$.- El muestreo para la estructura de tallas fue bietápico. En la primera etapa, se seleccionó una muestra aleatoria de $n$ viajes con sus respectivos lances y distribuciones de tallas. En la segunda etapa, para cada lance, se sacó una muestra de 200 ejemplares (individuos) y se calcularon el promedio y la desviación estándar de las tallas. Estas dos etapas se repitieron $k$ veces para, finalmente, calcular intervalos de confianza del promedio y la desviación estándar.

\section{Estimación del porcentaje de ocurrencia de juveni- les en las capturas de anchoveta}

Simulación de la población y cálculo de los parámetros poblacionales.- Para una población de $N$ viajes de pesca en el día, primero se simuló el número de lances en cada viaje. Luego, se simuló el número total de viajes con ocurrencia de juveniles y se seleccionó aleatoriamente, de la población de $N$ viajes, cuáles tuvieron ocurrencia de juveniles. En los viajes con ocurrencia de
Tabla 2. Objetivos, indicadores calculados a partir de los datos del PBP

\begin{tabular}{ll}
\hline Objetivo & Indicadores \\
\hline Talla de las capturas & Promedio y desviación estándar
\end{tabular}

Ocurrencia de juveniles $\quad$ Porcentaje de ocurrencia

$\begin{array}{ll}\text { Descartes por exceso de captura } & \begin{array}{l}\text { Porcentaje de viajes con } \\ \text { descartes por exceso, promedio } \\ \text { y desviación estándar del } \\ \text { volumen descartado }\end{array}\end{array}$

Porcentaje de viajes con

Descartes de juveniles descartes de juveniles, promedio y desviación estándar del volumen descartado

Porcentaje de viajes con Captura incidental captura incidental, promedio y desviación estándar del volumen de captura incidental

Profundidad de los cardúmenes Promedio y desviación estándar

Duración de los viajes Promedio y desviación estándar

Captura por unidad de esfuerzo Promedio y desviación estándar

juveniles, se simuló cuántos de sus lances tuvieron ocurrencia de juveniles. Finalmente, se calculó la proporción de lances con juveniles del total de lances de los $N$ viajes.

Muestreo para cada tamańo de muestra $\boldsymbol{n}$.- Primero se sacó una muestra aleatoria de $n$ viajes con sus respectivos lances y ocurrencias de juveniles. Luego, se calculó la proporción de lances con ocurrencia de juveniles del total de lances en los $n$ viajes. Estos pasos se repitieron $k$ veces para, finalmente, calcular intervalos de confianza de la proporción de lances con ocurrencia de juveniles.

\section{Estimación del porcentaje de viajes con descartes de anchoveta por exceso de captura, con descartes de juveniles, con captura incidental, y estimación de los volúmenes de descarte y de captura incidental}

Tanto para el descarte por exceso de captura, descarte por juveniles y la captura incidental, el procedimiento es similar y se describe a continuación para los tres objetivos.

Simulación de la población y cálculo de los parámetros poblacionales.- Para la población de $N$ viajes de pesca en el año, primero se simuló el número de viajes con descarte por exceso de captura (o con descarte de juveniles o con captura incidental). Para cada viaje con descarte (o captura incidental), se simuló el volumen descartado (o de captura incidental). Luego, se calcularon la proporción de viajes con descarte por exceso de captura (o con descarte de juveniles o con captura incidental), el promedio y la desviación estándar de volumen descartado (o de captura incidental).

Muestreo para cada tamaño de muestra $\boldsymbol{n}$.- Primero se sacó una muestra aleatoria de $n$ viajes. Luego se calcularon la 
proporción de viajes con descarte por exceso de captura (o con descarte de juveniles o con captura incidental), el promedio y la desviación estándar de volumen descartado (o de captura incidental). Estos pasos se repitieron $k$ veces para, finalmente, calcular intervalos de confianza de la proporción de viajes con descarte por exceso de captura (o con descarte de juveniles o con captura incidental), del promedio y de la desviación estándar de volumen descartado (o de captura incidental).

\section{Estimación de la profundidad de los cardúmenes de anchoveta}

Simulación de la población y cálculo de los parámetros poblacionales.- Para la población de $N$ viajes de pesca en el mes/año, primero se simuló el número de lances en cada viaje. Luego, para cada lance, se simuló la profundidad promedio del cardumen. Finalmente, se calcularon la media y la desviación estándar de la profundidad de los cardúmenes calados.

Muestreo para cada tamaño de muestra $\boldsymbol{n}$.- Primero se sacó una muestra aleatoria de $n$ viajes con sus respectivos lances. Luego, se calcularon el promedio y la desviación estándar de la profundidad de los cardúmenes. Estos pasos se repitieron $k$ veces para, finalmente, calcular intervalos de confianza del promedio y de la desviación estándar de la profundidad de los cardúmenes.

\section{Estimación de la duración de los viajes y de la CPUE}

Simulación de la población y cálculo de los parámetros poblacionales.- Para la población de $N$ viajes de pesca en el mes/ ańo, primero se simuló la duración (o la CPUE, respectivamente) de cada viaje. Luego, se calcularon la media y la desviación estándar de la duración o CPUE de los viajes.

Muestreo para cada tamaño de muestra $n$.- Primero se sacó una muestra aleatoria de $n$ viajes. Luego, se calcularon el promedio y la desviación estándar de la duración o CPUE de los viajes de la muestra. Estos pasos se repitieron $k$ veces para, finalmente, calcular intervalos de confianza del promedio y de la desviación estándar de la duración o CPUE de los viajes.

En todos los casos, para comparar los indicadores poblacionales y muestrales, se calcularon los ECM para el promedio y la desviación estándar de las variables, dividiendo cada uno entre su respectivo valor máximo para tener una variable continua de 0 a 1. Luego, se eligió el mínimo $n$ a partir del cual la variación en ambos ECM (del promedio y de la desviación estándar) fuera menor a 0.05 .

Antes de seleccionar el tamaño de muestra óptimo para cada indicador, se probó si algún parámetro de los utilizados en la simulación (Anexo 1) tuvo un efecto significativo sobre el tamaño de muestra. Para ello se utilizó el test de Kruskal-Wallis. Para hallar qué valores tuvieron una diferencia significativa frente a los otros, se utilizó el test de Wilcoxon (Siegel 1956). Para esos tests se utilizaron las librerías stats (R Core Team 2014) y exactRankTests (Hothorn y Hornik 2013) del software R. Para poder asegurar una estimación precisa en diversas condiciones, se calculó el tamańo de muestra para las condiciones más conservadoras entre los datos históricos, es decir, para los valores de los parámetros que necesitaron un tamaño de muestra significativamente más grande que el resto. El tamaño óptimo fue la mediana de los tamańos de muestra hallados bajo estas condiciones.

\section{Resultados}

Para todos los indicadores se observó que a medida que el tamaño de muestra aumentaba, la estimación de los indicadores se estabilizó: los intervalos de confianza fueron más pequeños y las estimaciones fueron más precisas. Como ejemplo podemos observar en la Figura 1 la estimación de los descartes por juveniles, donde podemos observar los intervalos de confianza y el ECM para la proporción de descartes, el promedio y la
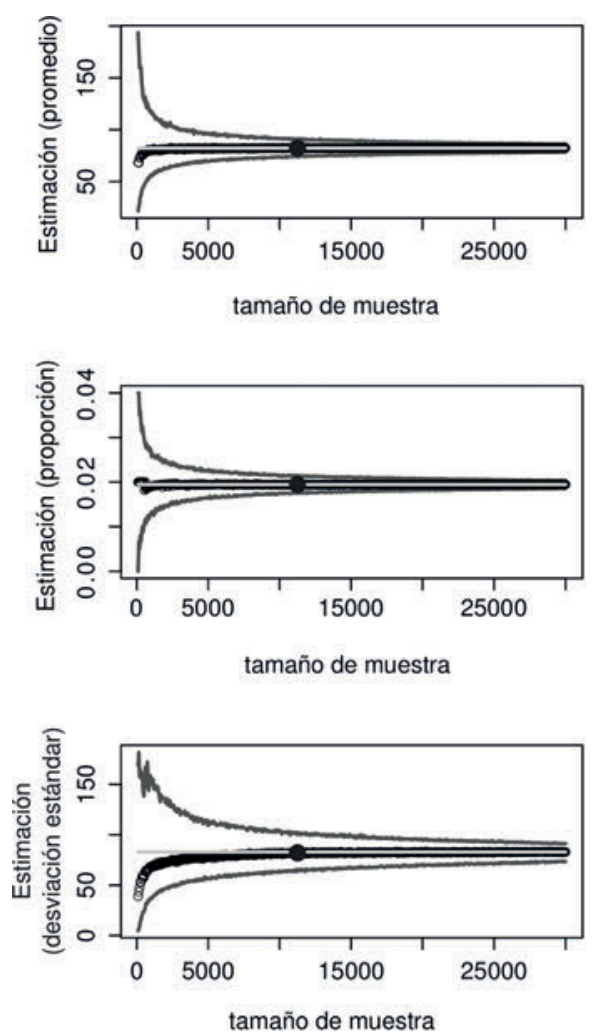
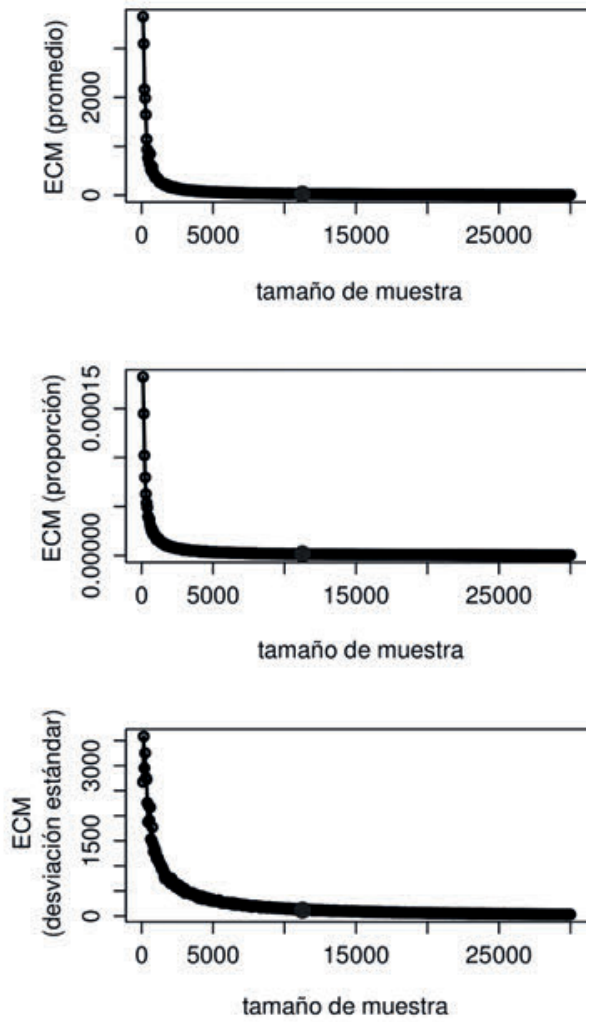

Figura 1. Cálculos de bandas de confianza y error cuadrático medio (ECM) para los descartes de juveniles, para un ejemplo donde $\mathrm{N}=57000$. A la izquierda: estimaciones del promedio (arriba), la proporción (en medio) y la desviación estándar (abajo) para cada tamaño de muestra. El valor verdadero está representado por una línea horizontal en gris claro. Las bandas de confianza están en gris oscuro. Los valores estimados se representan por circunferencias negras. A la derecha: error cuadrático medio para el promedio (arriba), la proporción (en medio) y la desviación estándar (abajo), para cada tamaño de muestra. En todos los casos, el valor en círculo negro indica el valor óptimo para esa réplica. 
desviación estándar del volumen descartado para las diferentes realizaciones de las simulaciones y tomas de muestra, mientras el tamaño de muestra $n$ aumenta. Las figuras análogas para los otros indicadores se encuentran en el Anexo 2. A continuación describimos los resultados obtenidos para cada variable.

Estructura de tallas.- Para la estructura de tallas, el tamaño $\mathrm{N}$ de la población de viajes tuvo un efecto significativo sobre el tamaño de muestra óptimo $\left(\mathrm{p}<10^{-5}\right)$. Los tamaños calculados para el mayor valor de N, 9300, fueron significativamente superiores a los calculados con los otros valores de $\mathrm{N}(\mathrm{p}<0.01)$. La mediana del tamaño de muestra necesario para $\mathrm{N}=9300$ fue 975 viajes en el mes.

Ocurrencia de juveniles.- El tamaño $\mathrm{N}$ de la población también tuvo un efecto significativo sobre el tamańo de muestra para la estimación de la ocurrencia de juveniles $\left(\mathrm{p}<10^{-15}\right)$. Para el mayor valor de N, 1500 viajes diarios, se obtuvieron tamaños de muestra más grandes $\left(\mathrm{p}<10^{-15}\right)$. La mediana del tamaño de muestra necesario para $\mathrm{N}=1500$ fue 180 viajes por día.

Descartes por exceso.- El tamaño de la población de viajes, la proporción de viajes con descartes por exceso y la media del volumen descartado fueron los tres parámetros que presentaron efectos significativos para la estimación del tamaño de muestra de viajes ( $\mathrm{p}<10^{-3}$ para las tres variables). Con el menor tamaño de la población, $\mathrm{N}=30000$, se obtuvo menores tamaños de muestra que con los otros dos tamańos poblacionales $\left(\mathrm{p}<10^{-3}\right)$. Asimismo, con las menores proporciones de viajes con descarte se necesitó mayores tamańos de muestra para una correcta estimación de la proporción y del volumen descartado $\left(\mathrm{p}<10^{-5}\right)$. A su vez, cuando el volumen de descarte poblacional fue menor, se necesitó un mayor número de viajes muestreados para estimarlo de manera precisa $\left(\mathrm{p}<10^{-4}\right)$. Así, las condiciones más difíciles fueron aquellas de tamaño poblacional alto, bajas proporciones de viajes con descarte y bajos volúmenes de descarte poblacional. Para estas condiciones, se calculó un tamaño de muestra óptimo igual a 5150 viajes en el año.

Descartes de juveniles.- Para la estimación de descartes de juveniles, el tamaño de la población tuvo un efecto significativo en el cálculo del tamaño de muestra óptimo ( $p<0.05)$. El tamaño de muestra fue mayor para los dos tamaños de poblaciones más grandes $(\mathrm{N}=38000$ y 57000; $\mathrm{p}<0.01)$. El escenario más conservador fue aquel con esos dos tamańos de poblaciones, para el cual obtuvimos una mediana del tamaño de muestra igual a 11220 viajes en el año (Fig. 1).

Captura incidental.- Para las estimaciones de captura incidental, la media del volumen de captura incidental tuvo un efecto significativo sobre el tamańo de muestra calculado $\left(\mathrm{p}<10^{-5}\right)$. Un menor volumen de captura incidental poblacional requirió un mayor tamańo de muestra para poder estimarlo con precisión. Para esas condiciones obtuvimos una mediana de 16000 viajes de pesca al ańo como tamaño de muestra.

Profundidad de los cardúmenes.- Tanto a escala mensual como a escala anual se encontraron diferencias significativas en el tamaño de muestra para tamaños de poblaciones distintas ( $\mathrm{p}$ $<10^{-13}$ y $\mathrm{p}<0.01$, respectivamente). En la escala mensual, para el tamaño de población más grande, 9300, se necesitó muestras más grandes para estimar la profundidad de los cardúmenes ( $\mathrm{p}$ $<10^{-7}$ ). La mediana calculada para $\mathrm{N}=9300$ fue 975 viajes mensuales (Fig. 2.5a, Anexo 2). En la escala anual, el tamaño de población más pequeño requirió muestras significativamente menores a las requeridas con los otros tamaños de población ( $\mathrm{p}$ $<0.01$ ). Por ello se calculó el tamaño de muestra para los dos tamaños de población más grandes, 38000 y 57000; el valor calculado fue 2050 viajes anuales.

Duración de los viajes.- Tanto a escala mensual como a escala anual se encontraron diferencias significativas en el tamaño de muestra para tamańos de poblaciones distintas $\left(\mathrm{p}<10^{-12} \mathrm{y}\right.$ $\mathrm{p}<0.01$, respectivamente). En la escala mensual, para los dos tamaños poblacionales más grandes, 6700 y 9300, se necesitó muestras más grandes para estimar la duración $\left(\mathrm{p}<10^{-13}\right)$. Por ello, se calculó el tamaño de muestra sólo para estos dos tamaños de poblaciones. La mediana calculada fue 1038 viajes mensuales (Fig. 2.6a, Anexo 2). En la escala anual, el tamaño de población más grande (57000) requirió muestras significativamente mayores a las requeridas con los otros tamaños de población ( $\mathrm{p}<0.01)$. Por ello se calculó el tamaño de muestra para aquel tamaño poblacional; el valor calculado fue 2150 viajes anuales.

Captura por unidad de esfuerzo.- Para la CPUE, no hubo diferencias significativas del tamaño de la población. Se calculó la mediana para todas las simulaciones, lo que dio un valor igual a 3050 viajes por año.

Los valores de tamaño de muestra óptimo para cada indicador se muestran en la Tabla 3.

\section{Discusión}

En este trabajo, obtuvimos tamaños de muestra óptimos para diversos indicadores estimándolos a partir de muestras de viajes del PBP. Los cálculos fueron realizados a partir de simulaciones que recrearon condiciones diversas observadas históricamente por el PBP.

Los tamaños de muestra obtenidos para cada variable son muy distintos (Tabla 3), lo cual refleja que cada variable -y entonces cada objetivo de monitoreo o investigación acompañado de una escala temporal específica- tiene un tamaño de muestra asociado. Por ejemplo, para la escala anual, los objetivos que requirieron tamaños de muestra más grandes fueron la estimación de la captura incidental (incidencia y volumen) y la estimación de descartes requiere un tamaño de muestra mayor que todos los otros objetivos. La tolerancia máxima de volumen de captura incidental es 20\% de los desembarques; sobrepasar este nivel de tolerancia llevaría a penalidades económicas para las embarcaciones pesqueras. La abundancia y accesibilidad de la anchoveta

Tabla 3. Tamaños de muestra óptimos para cada indicador a las escalas estudiadas.

\begin{tabular}{|c|c|c|c|}
\hline \multirow{2}{*}{ Objetivos } & \multicolumn{3}{|c|}{ Escala } \\
\hline & Diaria & Mensual & Anual \\
\hline Estructura de tallas & - & 975 & - \\
\hline Ocurrencia de juveniles & 180 & - & - \\
\hline Descartes por exceso & - & - & 5150 \\
\hline Descartes de juveniles & - & - & 11220 \\
\hline Captura incidental & - & - & 16000 \\
\hline Profundidad de cardúmen & - & 975 & 2050 \\
\hline Duración del viaje & - & 1038 & 2150 \\
\hline CPUE & - & - & 3050 \\
\hline
\end{tabular}


harían difícil que se llegue a este tope en la práctica (corroborado por los datos en el Anexo 1). Al existir una incidencia débil de captura incidental es más difícil estimar su volumen y por ello requiere un mayor número de viajes a muestrear.

El segundo objetivo en tamaño de muestra es el de estimación de descartes por juveniles (incidencia y volumen). Como para la captura incidental, el número de viajes con ocurrencia de juveniles es muy bajo, por lo que se requiere un alto número de viajes observados para la estimación. La captura de juveniles de anchoveta en una magnitud superior a la tolerancia establecida ( $10 \%$ en número) así como sus descartes están prohibidos y penados, y su registro puede generar vedas locales a muy corto plazo (Arias Schreiber et al. 2011). A diferencia de la captura incidental, la captura de juveniles es más probable puesto que las embarcaciones podrían encontrarse en zonas con predominancia de juveniles y/o realizar calas compuestas por adultos y juveniles (Fablet et al. 2012). Todo esto podría originar un sesgo en los registros de los observadores: por más que el observador de IMARPE no cumpla labores de fiscalización, su sola presencia podría inhibir a los pescadores de pescar en áreas con sabida ocurrencia de juveniles y de cometer descartes. Esta situación es conocida como "efecto observador" (Liggins et al. 1997, Babcock y Pikitch 2003). Para cuantificar este sesgo, podrían realizarse simulaciones en base a supuestos sobre el comportamiento de los pescadores bajo diversos escenarios de presencia de juveniles, lo que estaría fuera del alcance de este trabajo. Muy por el contrario, las variables que son comúnmente registradas dentro del procedimiento de colección de información por el PBP, como el esfuerzo, la CPUE o la profundidad de los cardúmenes, requieren un menor tamaño de muestra.

Para la mayoría de indicadores, el tamaño de la población jugó un papel importante en el tamańo de muestra hallado. Es lógico que cuando el tamaño de la población aumenta, también debe hacerlo el de la muestra para que ésta pueda seguir siendo representativa; aunque la relación no es lineal sino logarítmica, lo que implica además que para tamaños de población muy grandes, el tamaño de muestra casi no varía. El tamaño de muestra hallado también depende de la variabilidad existente. Pusimos como condición que el ECM (relativo) de la desviación estándar también sea menor a 0.05 . Si bien la desviación estándar no debe ser necesariamente igual a la poblacional, esperamos que, en todo caso, llegue a un umbral de proximidad aceptable. En muchos casos fue la desviación estándar lo que requirió mayor tamaño de muestra para poder estabilizarse (e.g. Fig. 2.7, Anexo 2). Con el criterio utilizado, se garantiza entonces una reducción de la varianza y por lo tanto estimaciones más precisas.

En cuanto a recomendaciones prácticas para fijar un único tamaño de muestra para todos los objetivos, recomendamos tomar el máximo tamaño obtenido en las diferentes escalas temporales. Así se logran cubrir todos los objetivos. De esta manera, 180 viajes al día permitirían una estimación precisa de la ocurrencia de juveniles diaria, 1038 viajes al mes asegurarían una estimación de los parámetros de media y desviación estándar de las tallas, profundidad de la anchoveta y duración de los viajes, y 16000 viajes anuales permitirían una adecuada estimación de descartes por exceso, de juveniles, captura incidental, profundidad de los cardúmenes, duración de los viajes y CPUE anuales. Con 180 viajes al día por 20 días de trabajo al mes que se le suele asignar a los observadores por los 6 meses al año que se suele pescar anchoveta, se cumplirían con los tamaños de muestra en las tres escalas temporales (21600 viajes).

En este estudio se estimó un tamaño de muestra por cada objetivo por tres razones principales: (1) los indicadores de interés estaban a diferentes escalas temporales; (2) el orden de prioridad de cada objetivo puede variar con el tiempo, por lo que fijar una ponderación para cada uno (Miller et al. 2007) puede ser contraproducente; y (3) quisimos que la incertidumbre sobre cada variable (relacionada a valores en los parámetros para la simulación) no afecte las simulaciones para las otras variables. Respecto al último punto, una alternativa metodológica a lo mostrado en este estudio sería simular la población de anchovetas y de otros recursos con sus respectivas tallas, simular los lances y su composición en cada uno de los viajes pesqueros en el año hasta alcanzar las cuotas establecidas por el Estado, y elegir aleatoriamente los viajes que son observados. A partir de ello, calcular los valores poblacionales de los indicadores y compararlos con los calculados por la submuestra observada. Es una metodología válida; sin embargo, cada una de las etapas de simulación reposa sobre supuestos que, si alguno se aleja de la realidad, sesgan todos los cálculos. En este estudio nos valemos para las simulaciones solamente de los datos históricos del programa Bitácoras de Pesca. Al ser valores calculados a partir de muestras, estos datos también están sujetos a error. No obstante, al haber analizado varios escenarios posibles, podemos considerar que los resultados son robustos a posibles errores. Además esta metodología queda expedita para estimar un nuevo tamaño óptimo de muestra del PBP ante la inclusión de nuevos objetivos de monitoreo e investigación.

En un escenario ideal, cada barco debería zarpar con un observador a bordo. En una pesquería tan dinámica y tan grande como la de la anchoveta peruana (en la última década llegó a un pico de -1200 embarcaciones operativas por día; Fréon et al. 2008), esta disposición sería logísticamente muy complicada. Por ello, el presente trabajo consistió en la estimación de un tamaño de muestra óptimo de viajes observados para una estimación precisa de diversos indicadores de monitoreo y manejo. La estimación de estos indicadores fue realizada de manera global, considerando muestreos aleatorios simples, a excepción de aquel de la estructura de tallas, que tuvo una segunda etapa de selección de anchovetas. En el caso de que el objetivo sea estimar indicadores espacializados, o tener estimaciones precisas para cada segmento de flota, lo óptimo sería realizar un muestreo aleatorio multietápico (e.g. Gavaris \& Gavaris 1983, Montenegro Silva 2009, para desembarques), tomando en cuenta componentes espaciales (en base a los puertos de embarque y desembarque, por ejemplo) y los tipos de embarcaciones, obteniendo un tamaño de muestra por puerto y por tipo de embarcación.

Para la realización de las simulaciones se consideró que los viajes muestreados eran seleccionados aleatoriamente. En la práctica, participar en el programa es voluntario para las embarcaciones. Esta condición introduce un sesgo a las estimaciones de los indicadores. Lo ideal sería que no se tuviese que depender de la buena voluntad de los patrones de las embarcaciones, o que exista "suficiente buena voluntad como para permitir la selección aleatoria de las embarcaciones de la flota entera" (Cotter 2002). En ese caso se haría un muestreo multietápico que seleccione en una primera etapa a las embarcaciones y en una segunda etapa a 
los viajes (o días de viaje, que no es muy distinto en la pesquería de anchoveta ya que los viajes duran -24 horas). En el contexto actual, para reducir el sesgo, se podría considerar una tasa de no respuesta para estimar un número mayor de embarcaciones a muestrear, para luego seleccionarse aleatoriamente las embarcaciones invitándoles a participar en el programa (Hansen y Hurwitz 1946). Y si por ejemplo, las embarcaciones voluntarias suelen ser las que tienen bajos niveles de descarte, incrementar el número de embarcaciones en la muestra podría incluir más embarcaciones que son más típicas en la pesquería (Hall 1999). Otra alternativa sería hacer una lista de los posibles sesgos de la muestra voluntaria (Babcock y Pikitch 2003) y establecer una serie de condiciones que deban tener embarcaciones para considerarlas "representativas" y que puedan ser aceptadas como parte de la muestra.

En cuanto al "efecto observador", este se podría medir, por ejemplo, al probar si las declaraciones de descartes realizadas en puerto cuando no hay observador a bordo son significativamente menores a aquellas registradas en viajes con observadores para esas mismas embarcaciones. Lo mismo con la presencia de juveniles. Para hacer la comparación, habría que eliminar primero el efecto de condiciones ambientales para ambos grupos.

Respecto a los tamaños de muestras para otras especies objetivo en la pesquería pelágica de cerco, dado que hay un mayor número de embarcaciones dedicadas a la pesca de anchoveta, y asumiendo un comportamiento similar en la distribución de las variables de interés, el número de observadores necesarios para registrar viajes anchoveteros debería ser más que suficiente para garantizar una precisa estimación de estos mismos indicadores para otras especies. Si en la práctica no se puede cumplir siempre con el tamaño de muestra recomendado, los resultados obtenidos en el presente trabajo resultan muy útiles para comprender en qué condiciones se necesitan tamaños de muestra más grandes y en cuáles no; así como el nivel de imprecisión que acompañaría las estimaciones de los indicadores.

\section{Agradecimientos}

Gracias sinceras a Enrique Ramos y Pablo Marín por la preparación de los datos históricos que sirvieron de inputs para los parámetros de las simulaciones; muchísimas gracias por su disponibilidad. Gracias también a Gersson Román, por la información histórica sobre la cobertura del PBP. Agradecemos a los bitacoreros, sin cuya labor, todo esto no sería posible.

\section{Literatura citada}

Andrew N.L. \& Y. Chen , 1997. Optimal sampling for estimating the size structure and mean size of abalone caught in a New South Wales fishery. Fish. Bull. 95, 403-413.

Aranda, M. 2009. Developments on fisheries management in Peru: The new individual vessel quota system for the anchoveta fishery. Fish. Res. 96, 308-312. Doi: http://dx.doi.org/10.1016/j. fishres.2008.11.004

Arias Schreiber M., M. Niquen \& M. Bouchon. 2011. Coping Strategies to Deal with Environmental Variability and Extreme Climatic Events in the Peruvian Anchovy Fishery. Sustainability 3, 823-846. Doi: http://dx.doi.org/10.3390/su3060823

Avadí A., P. Fréon \& I. Quispe. 2014. Environmental assessment of Peruvian anchoveta food products: is less refined better? Int. J. Life Cycle Assess. 19, 1276-1293. Doi: http://dx.doi. org/10.1007/s11367-014-0737-y
Babcock E.A. \& E.K. Pikitch. 2003. How much observer coverage is enough to adequately estimate bycatch? Pew Institute for Ocean Science and Oceana, $36 \mathrm{p}$.

Chavez F., A. Bertrand, R. Guevara-Carrasco, P. Soler \& J. Csirke. 2008. The northern Humboldt Current System: Brief history, present status and a view towards the future. Progress in Oceanography 79, 95-105. Doi: http://dx.doi. org/10.1016/j.pocean.2008.10.012

Cotter A.J.R., G. Course, S.T. Buckland \& C. Garrod. 2002. A PPS sample survey of English fishing vessels to estimate discarding and retention of North Sea cod, haddock, and whiting. Fisheries Research 55, 25-35. Doi: http://dx.doi. org/10.1016/S0165-7836(01)00306-X

Fablet R., P. Gay, S. Peraltilla, R. Castillo \& A. Bertrand. 2012. Bagsof-Features for fish school cluster characterization in pelagic ecosystems: application to the discrimination of juvenile and adult anchovy clusters off Peru. Canadian Journal of Fisheries and Aquatic Sciences 69, 1329-1339. Doi: http://dx.doi. org/10.1139/f2012-064

Freón P., M. Bouchon, C. Mullon, C. García, \& M. Ñiquen. 2008. Interdecadal variability of anchoveta abundance and overcapacity of the fishery in Peru. Progress in Oceanography 79, 401-412. Doi: http://dx.doi.org/10.1016/j. pocean.2008.10.011

Gavaris S. \& C. Gavaris. 1983. Estimation of catch at age and its variance for groundfish stocks in the Newfoundland region, $\mathrm{p}$. 178-182. En W. G. Doubleday y D. Rivard (ed.). Sampling commercial catches of marine fish and invertebrates. Canadian Special Publication of Fisheries and Aquatic Publication speciale canadienne des sciences halieutiques Sciences 66.

Hall M.A. 1999. Estimating the ecological impacts of fisheries: what data are needed to estimate bycatches? p. 175-184. En C. P. Nolan (ed.) Proceedings of the International Conference on Integrated Fisheries Monitoring. Sydney, Australia, $1-5$ Febrero 1999.

Hansen M. \& W. Hurwitz. 1946. The problem of non response in sampling surveys. Jour. Amer. Stat. Assoc. 41, 517-529. Doi: http://dx.doi.org/10.2307/2280572

Hothorn T. \& K. Hornik. 2013. exactRankTests: Exact Distributions for Rank and Permutation Tests. R package version 0.8-27. http://CRAN.R-project.org/package=exactRankTests

Liggins G.W., M.J. Bradley \& S.J. Kennelly. 1997. Detection of bias in observer-based estimates of retained and discarded catches from a multi species trawl fishery. Fisheries Research 32, 133-147. Doi: http://dx.doi.org/10.1016/S01657836(97)00053-2

Miller T.J., J.R. Skalski \& J.N. Ianelli. 2007. Optimizing a stratified sampling design when faced with multiple objectives. ICES Journal of Marine Science 64, 97-109. doi: 10.1093/icesjms/ $f_{s l O 13}$

Montenegro Silva C. 2009. Tamaños de muestra para estimar la estructura de tallas de las capturas de langostino colorado en la zona centro-norte de Chile: una aproximación a través de remuestreo. Latin american journal of aquatic research 37, 161-180.

R Core Team. 2014. R: A language and environment for statistical computing. R Foundation for Statistical Computing, Vienna, Austria. URL http://www.R-project.org/.

Siegel S. 1956. Nonparametric statistics for the behavioral sciences. McGraw-Hill, New York.

Smith M.H. \& S.J. Baird. 2005. Representativeness of past observer coverage, and future coverage required for estimation of New Zealand sea lion (Phocarctos hookeri) captures in the SQU 6T fishery. New Zealand Fisheries Assessment Report 2005/5.39 p.

Tveteras S., C.E. Paredes \& J. Peña Torres. 2011. Individual Vessel Quotas in Peru: Stopping the Race for Anchovies. Marine Resource Economics 26, 225-232. doi: http://dx.doi. org/10.5950/0738-1360-26.3.225 


\section{Anexo 1: Parámetros utilizados para las simulaciones.}

Estos parámetros fueron obtenidos a partir de información histórica del programa bitácoras de pesca (PBP). Tamaño de las poblaciones: Dado que el PBP sólo cubre una muestra de la flota, la información del número de viajes realizados a escalas diaria, mensual y anual fue obtenida de datos de desembarques.

Se utilizaron como escenarios valores aproximados al primer cuartil, la mediana y el tercer cuartil (Tabla S1), exceptuando la escala diaria, para la que se utilizó aproximaciones a la mediana, el tercer cuartil y el máximo, debido a que los valores de mínimo y primer cuartil eran muy pequeños para poder estimar correctamente los indicadores a partir de submuestras. Así, los tamańos de las poblaciones de viajes de la flota utilizados fueron: 270, 470 y 1500 para la escala diaria, 2300, 6700 y 9300 para la escala mensual y 30000, 38000 y 57000 para la escala anual.

\section{Estructura de tallas:}

- El número calas por viaje es generado utilizando una distribución de Poisson de parámetro $\lambda$ igual a 3 , siendo 3 la moda del número de calas por viaje.

- El número de anchovetas que se miden en cada cala fue fijado en 200, pues es el número de anchovetas muestreadas por observadores en cada cala.

- Las tallas de la población de anchovetas fueron simuladas con una distribución normal de parámetros $\mu$ igual a 14.19 y sd igual a 0.95 . Estos valores corresponden al promedio y desviación estándar obtenidos con datos históricos del PBP (en $\mathrm{cm}$ ). Posteriormente se probaron otros valores de $\mu \mathrm{y}$ $s d$, pero éstos no cambiaron los resultados de cálculo del tamaño de muestra.

\section{Ocurrencia de juveniles:}

- El número calas por viaje es generado utilizando una distribución de Poisson de parámetro $\lambda$ igual a 3 , siendo 3 la moda del número de calas por viaje.

- El número de viajes con ocurrencia de juveniles en la población de viajes es generado a partir de una distribución binomial que utiliza como parámetro $\boldsymbol{p}$ que asumió los siguientes valores: $0.05,0.167$ y 0.4644 , que fueron mínimo, mediana y máximo de proporción de viajes con juveniles obtenidos con datos del PBP. Para cada uno de estos valores se halló un tamaño de muestra de viajes.

- Para los viajes con ocurrencia de juveniles, se simula cuántas calas de esos viajes tenían ocurrencia de juveniles utilizando una distribución binomial de parámetro $\boldsymbol{p}$ igual a 0.25 , promedio obtenido con datos de PBP.

\section{Descartes de anchoveta por exceso de capturas:}

- El número de viajes con descarte por exceso es simulado a partir de una distribución binomial de parámetro $\boldsymbol{p}$ que asumió los siguientes valores: $0.06,0.09,0.12$ y 0.19 , que corresponden al mínimo, media, mediana y máximo de proporción de viajes con descartes por exceso obtenidos con datos del PBP.

- El volumen descartado en cada viaje es simulado utilizando una distribución lognormal de parámetros $\mu$ y $s d$ de valores $3.57,4$ y 4.44 y $0.92,0.88$ y 0.82 , respectivamente. Estos valores corresponden a las transformaciones (momentos aritméticos) de 54.28, 80.69 y 119.29 ( $\mu$ ) y $62.37,86.75$ y $117.31(s d)$. Los primeros tres valores corresponden al mínimo, mediana y máximo de volumen de descarte por exceso calculados con datos del PBP, y los siguientes tres valores son sus desviaciones estándar correspondientes, en toneladas métricas.

\section{Descartes de juveniles:}

- El número de viajes con descarte por juveniles es simulado a partir de una distribución binomial de parámetro $\boldsymbol{p}$ que asumió un valor igual a 0.02 , promedio obtenido con datos del PBP.

- El volumen descartado en cada viaje es simulado utilizando una distribución lognormal de parámetros $\mu$ y $s d$ de valores 4.08 y 0.88 , respectivamente. Estos valores corresponden a los momentos aritméticos de 87.059 y 93.926, respectivamente. Estos dos últimos valores corresponden al promedio y desviación estándar de volumen descartado por exceso de juveniles calculados con datos del PBP (en toneladas métricas).

\section{Captura incidental:}

- El número de viajes con captura incidental es simulado a partir de una distribución binomial de parámetro $\boldsymbol{p}$ que asumió un valor igual a 0.4613 , promedio obtenido con datos del PBP.

- El volumen de captura incidental en cada viaje es simulado utilizando una distribución lognormal de parámetros $\mu$ y $s d$ de valores $-6.3,1.81$ y 2.85 y $2.36,0.66$ y 0.92 , respectivamente. Estos valores corresponden a los momentos aritméticos de $0.03,7.6$ y 26.47 ( $\mu$ ) y $0.49,5.59$ y 30.64 (sd). Los primeros tres valores corresponden al mínimo, mediana y máximo de volumen de captura incidental calculados con datos del PBP, y los siguientes tres valores son sus desviaciones estándar correspondientes, en toneladas métricas.

Tabla S1. Estadísticas de mínimo, primer cuartil, mediana, tercer cuartil y máximo número de viajes realizados por la flota a escalas diaria, mensual y anual.

\begin{tabular}{lccccc}
\hline Escala & Mínimo & Primer cuartil & Mediana & Tercer cuartil & Máximo \\
\hline Diaria & 1 & 63 & 266 & 467 & 1499 \\
Mensual & 18 & 2367 & 6680 & 9319 & 24333 \\
Anual & 591 & 30767 & 37926 & 56381 & 75955 \\
\hline
\end{tabular}




\section{Profundidad de los cardúmenes:}

- El número de calas por viaje es generado utilizando una distribución de Poisson de parámetro $\lambda$ igual a 3 , siendo 3 la moda del número de calas por viaje.

- La profundidad de un cardumen (en metros) es simulada utilizando una distribución lognormal. Para la escala mensual, los valores de los parámetros $\mu$ y sd fueron 1.78 , 2.35 y 2.98 y $0.40,0.43$ y 0.20 , respectivamente; y correspondieron a los momentos aritméticos de 6.42, 11.51 y $20.18(\mu)$ y $2.69,5.19$ y $4.16(s d)$. Los primeros tres valores corresponden al mínimo, mediana y máximo de profundidad calculados con datos del PBP, y los siguientes tres valores son sus desviaciones estándar correspondientes. Para la escala anual, los valores de los parámetros $\mu$ y sd fueron $2.18,2.37$ y 2.54 y $0.36,0.38$ y 0.39 , respectivamente; y correspondieron a los momentos aritméticos de $9.4,11.5$ y 13.7 ( $\mu$ ) y $3.5,4.5$ y $5.5(s d)$. Los primeros tres valores corresponden al mínimo, mediana y máximo de profundidad calculados con datos del PBP, y los siguientes tres valores son sus desviaciones estándar correspondientes.

\section{Duración de los viajes:}

- La duración de los viajes (en horas) fue simulada utilizando una distribución lognormal. Para la escala mensual, los va- lores de los parámetros mu y sd fueron $2.52,2.91$ y 3.41 y $0.49,0.48$ y 0.61 , respectivamente; y correspondieron a los momentos aritméticos de $14.08,20.55$ y $36.54(\mu)$ y 7.33 , 10.49 y $24.58(s d)$. Los primeros tres valores corresponden al mínimo, mediana y máximo de duración calculados con datos del PBP, y los siguientes tres valores son sus desviaciones estándar correspondientes. Para la escala anual, los valores de los parámetros $\mu$ y $s d$ fueron $2.82,2.98$ y 3.18 y $0.48,0.47$ y 0.51 , respectivamente; y correspondieron a los momentos aritméticos de 18.85, 21.97 y 27.34 ( $\mu$ ) y 9.57 , 10.83 y $14.86(s d)$. Los primeros tres valores corresponden al mínimo, mediana y máximo de duración calculados con datos del PBP, y los siguientes tres valores son sus desviaciones estándar correspondientes.

Captura por unidad de esfuerzo

- La CPUE de los viajes fue simulada utilizando una distribución lognormal de parámetros mu y sd cuyos valores fueron 2.30 y 0.93 , respectivamente. Estos valores corresponden a los momentos aritméticos de 15.37 y 18.13 , respectivamente. Estos dos últimos valores corresponden al promedio y desviación estándar de CPUE calculados con datos del PBP.

\section{Anexo 2: Figuras de intervalos de confianza y error cuadrático medio de las muestras}
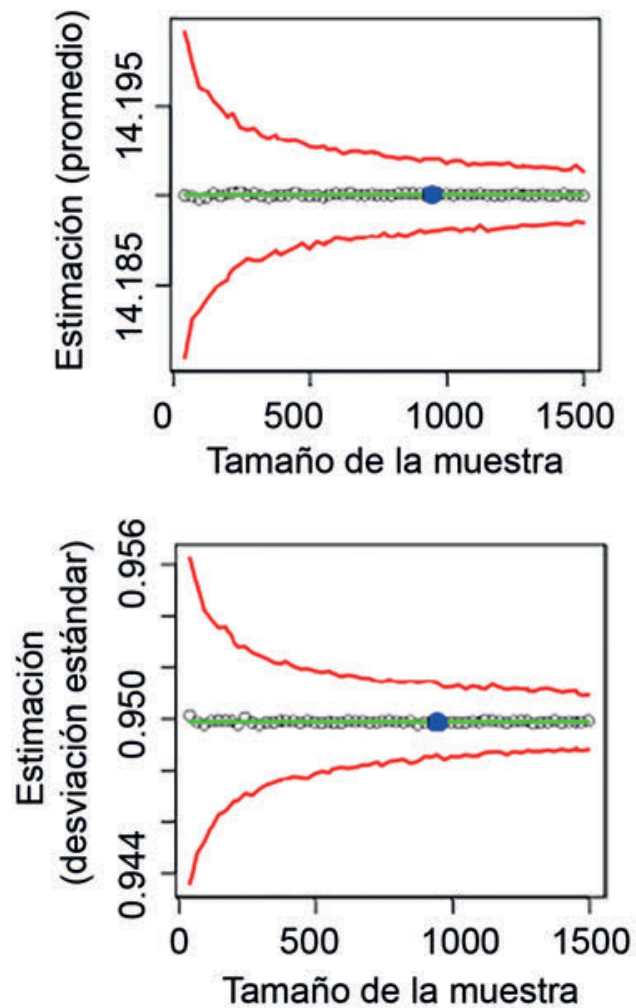
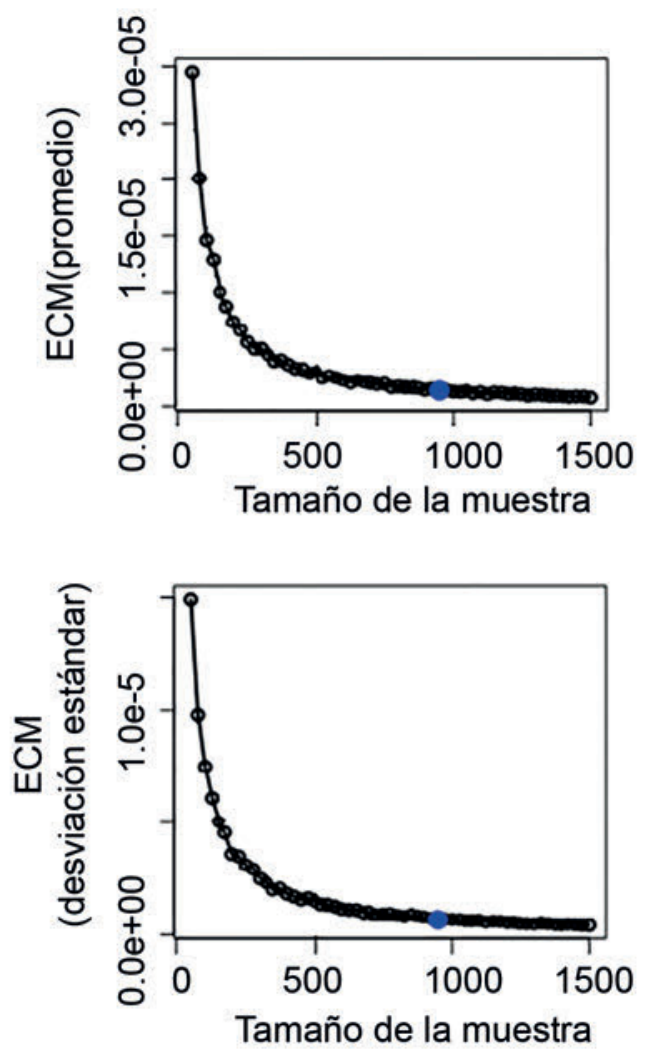

Figura 2.1. Cálculos de bandas de confianza y error cuadrático medio (ECM) para el promedio y la desviación estándar de las tallas de anchoveta, para un ejemplo donde $\mathrm{N}=9300$. A la izquierda: estimaciones del promedio (arriba) y la desviación estándar (abajo) para cada tamaño de muestra. El valor verdadero está representado por una línea horizontal verde. Las bandas de confianza están en rojo. Los valores estimados se representan por circunferencias negras. A la derecha: error cuadrático medio para el promedio (arriba) y la desviación estándar (abajo), para cada tamaño de muestra. En todos los casos, el valor azul indica el valor óptimo para esa réplica. 

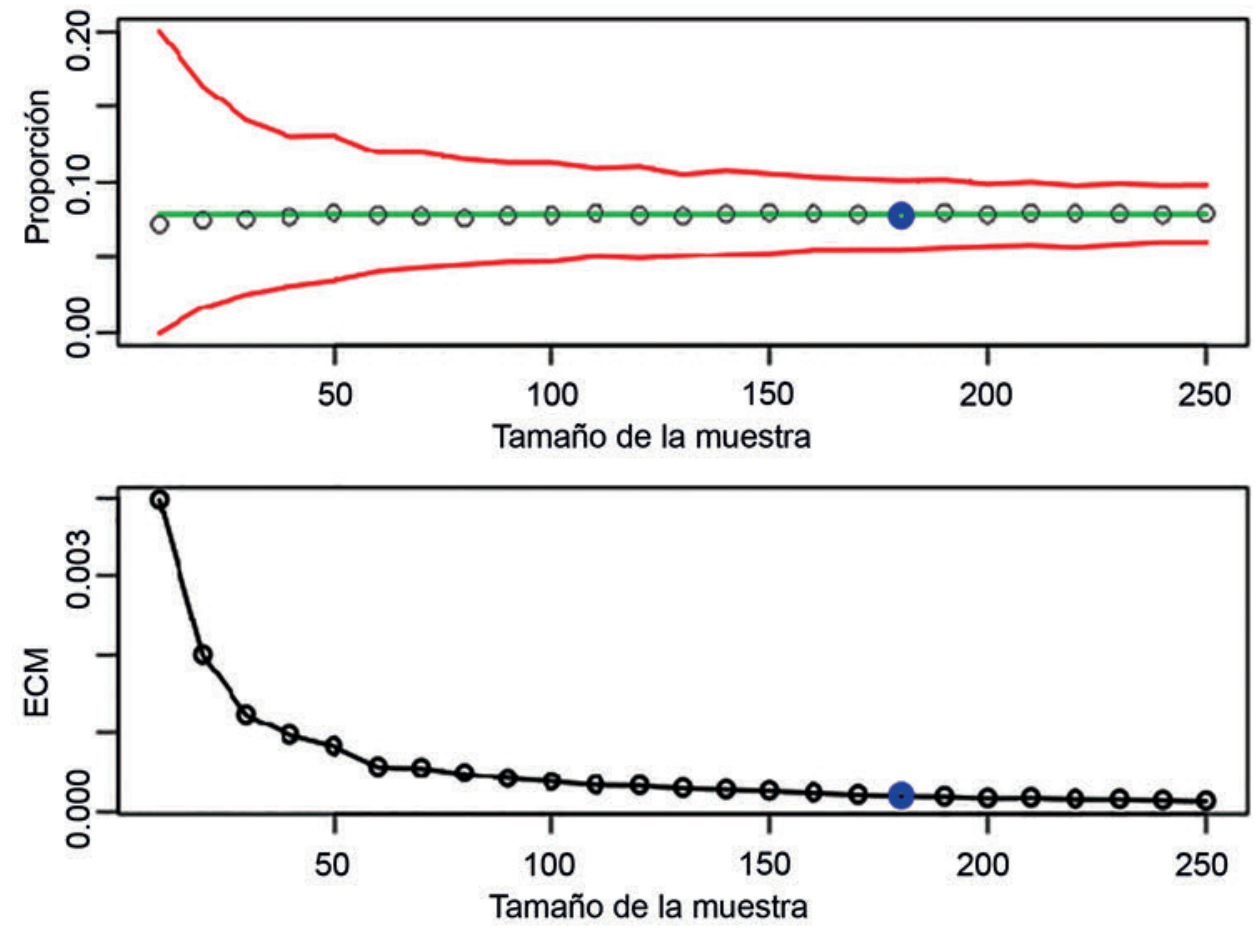

Figura 2.2. Cálculos de bandas de confianza y error cuadrático medio (ECM) para la proporción de juveniles, para un ejemplo donde $\mathrm{N}=$ 1500. Arriba: Estimaciones de la proporción de juveniles para cada tamaño de muestra. El valor verdadero está representado por una línea horizontal verde. Las bandas de confianza están en rojo. Los valores estimados se representan por circunferencias negras. Abajo: error cuadrático medio para la proporción de juveniles para cada tamaño de muestra. En los dos casos, el valor azul indica el valor óptimo para esa réplica.
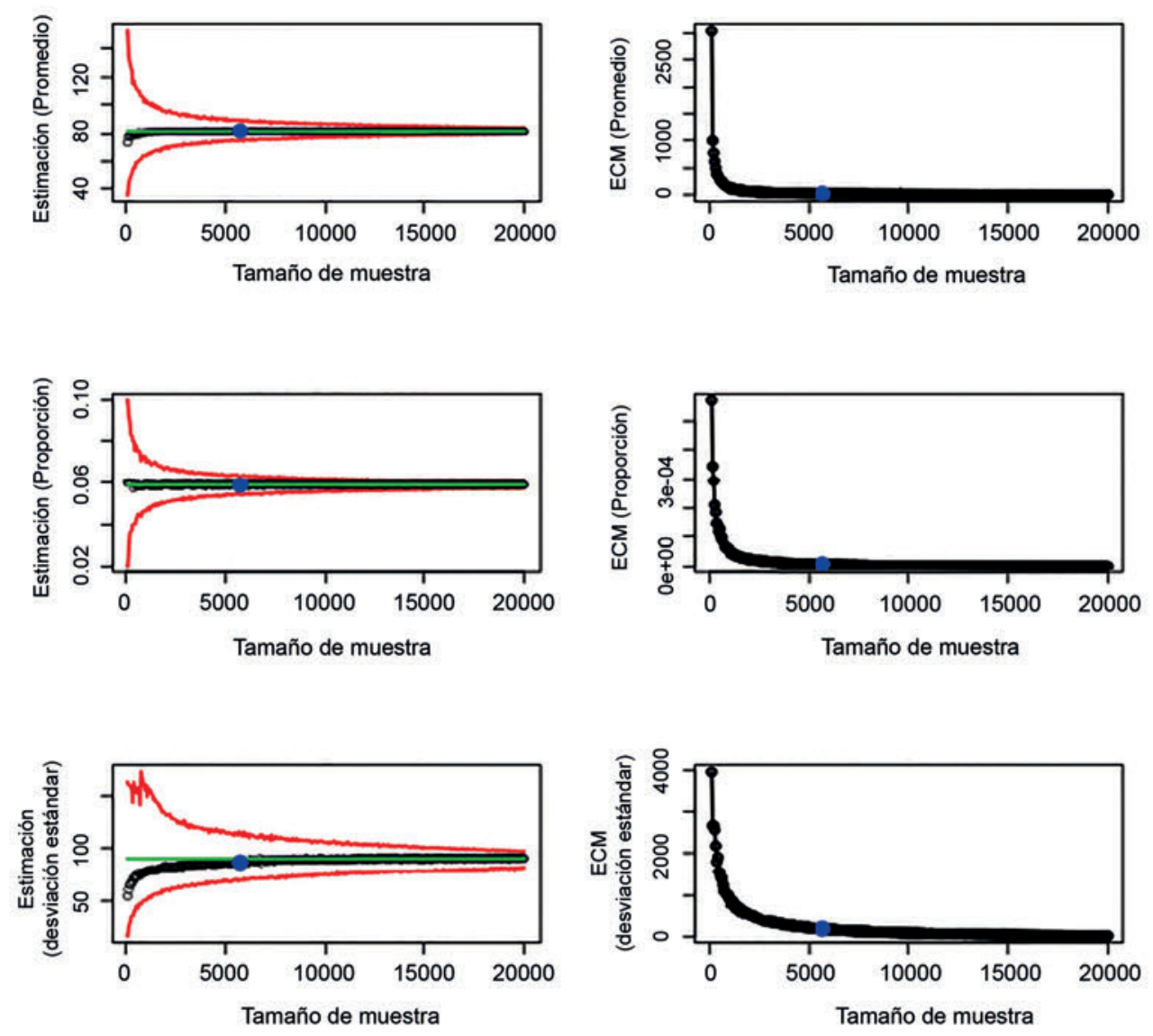

Figura 2.3. Cálculos de bandas de confianza y error cuadrático medio (ECM) para los descartes por exceso, para un ejemplo donde $\mathrm{N}=57000$, la proporción de viajes con descarte es igual a 0.06 y la media de descarte es 54 TN. A la izquierda: estimaciones del promedio (arriba), la proporción (en medio) y la desviación estándar (abajo) para cada tamaño de muestra. El valor verdadero está representado por una línea horizontal verde. Las bandas de confianza están en rojo. Los valores estimados se representan por circunferencias negras. A la derecha: error cuadrático medio para el promedio (arriba), la proporción (en medio) y la desviación estándar (abajo), para cada tamaño de muestra. En todos los casos, el valor azul indica el valor óptimo para esa réplica. 

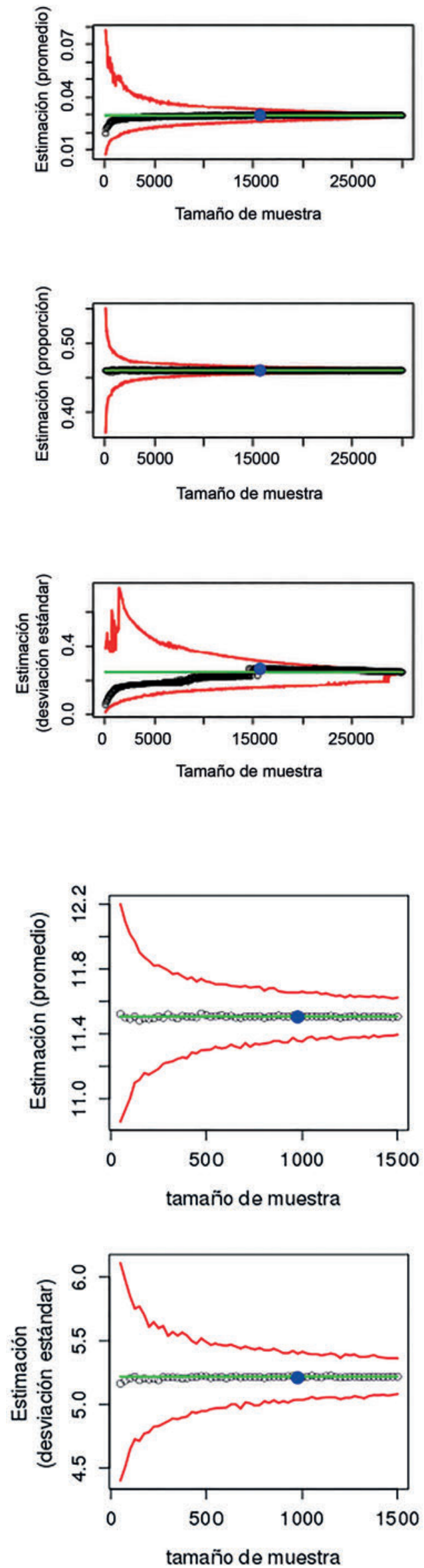
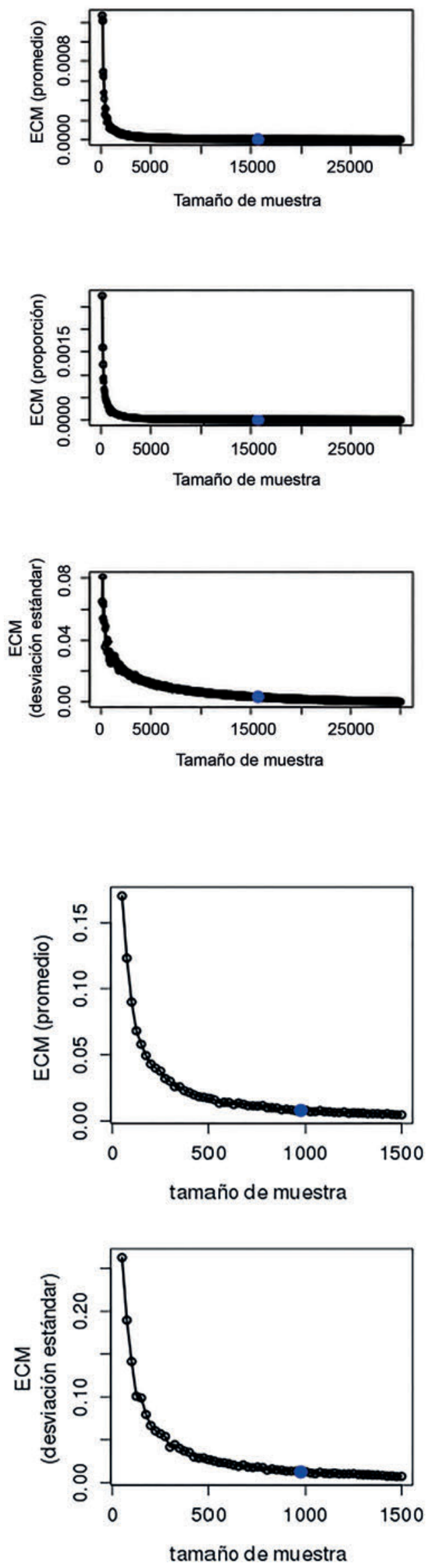

Figura 2.4. Cálculos de bandas de confianza y error cuadrático medio (ECM) para la captura incidental, para un ejemplo donde $\mathrm{N}=30000$ y la media de captura incidental es $30 \mathrm{~kg}$. A la izquierda: estimaciones del promedio (arriba), la proporción (en medio) y la desviación estándar (abajo) para cada tamaño de muestra. El valor verdadero está representado por una línea horizontal verde. Las bandas de confianza están en rojo. Los valores estimados se representan por circunferencias negras. Ala derecha: error cuadrático medio para el promedio (arriba), la proporción (en medio) y la desviación estándar (abajo), para cada tamaño de muestra. En todos los casos, el valor azul indica el valor óptimo para esa réplica.

Figura 2.5a. Cálculos de bandas de confianza y error cuadrático medio (ECM) para el promedio y la desviación estándar de la profundidad de los cardúmenes a escala mensual, para un ejemplo donde $\mathrm{N}=9300$. A la izquierda: estimaciones del promedio (arriba) y la desviación estándar (abajo) para cada tamaño de muestra. El valor verdadero está representado por una línea horizontal verde. Las bandas de confianza están en rojo. Los valores estimados se representan por circunferencias negras. A la derecha: error cuadrático medio para el promedio (arriba) y la desviación estándar (abajo), para cada tamaño de muestra. En todos los casos, el valor azul indica el valor óptimo para esa réplica. 

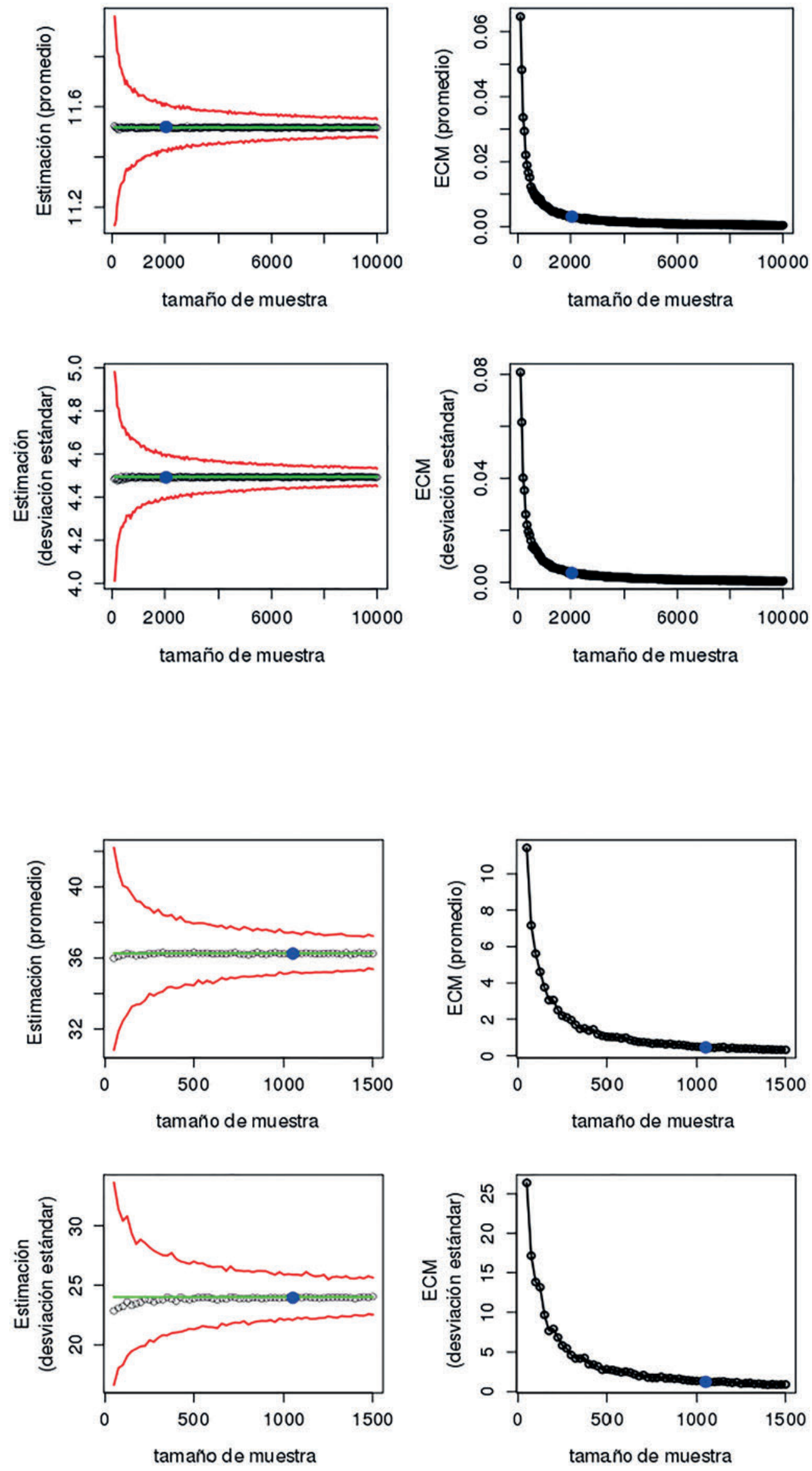

Figura 2.5b. Cálculos de bandas de confianza y error cuadrático medio (ECM) para el promedio y la desviación estándar de la profundidad de los cardúmenes a escala anual, para un ejemplo donde $\mathrm{N}=38000$. A la izquierda: estimaciones del promedio (arriba) y la desviación estándar (abajo) para cada tamaño de muestra. El valor verdadero está representado por una línea horizontal verde. Las bandas de confianza están en rojo. Los valores estimados se representan por circunferencias negras. Ala derecha: error cuadrático medio para el promedio (arriba) y la desviación estándar (abajo), para cada tamaño de muestra. En todos los casos, el valor azul indica el valor óptimo para esa réplica.

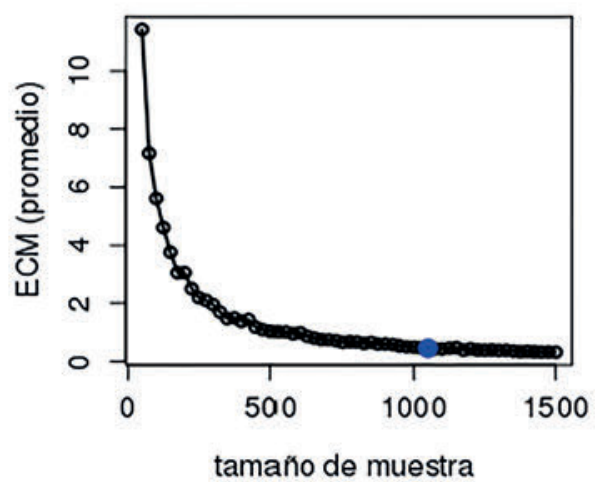

Figura 2.6a. Cálculos de bandas de confianza y error cuadrático medio (ECM) para el promedio y la desviación estándar de la duración del viaje a escala mensual, para un ejemplo donde $\mathrm{N}=9300$. A la izquierda: estimaciones del promedio (arriba) y la desviación estándar (abajo) para cada tamaño de muestra. El valor verdadero está representado por una línea horizontal verde. Las bandas de confianza están en rojo. Los valores estimados se representan por circunferencias negras. A la derecha: error cuadrático medio para el promedio (arriba) y la desviación estándar (abajo), para cada tamaño de muestra. En todos los casos, el valor azul indica el valor óptimo para esa réplica. 


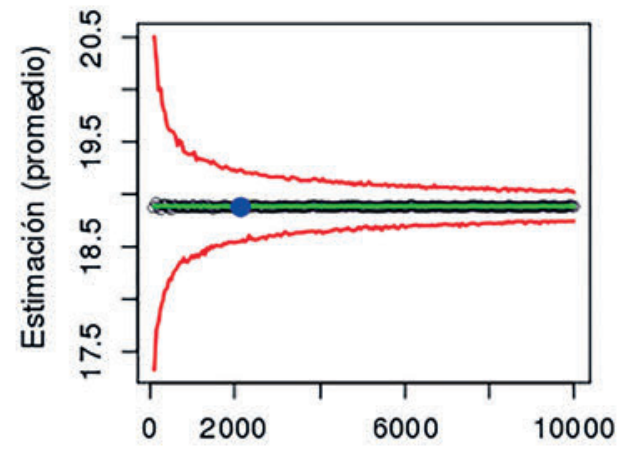

tamaño de muestra

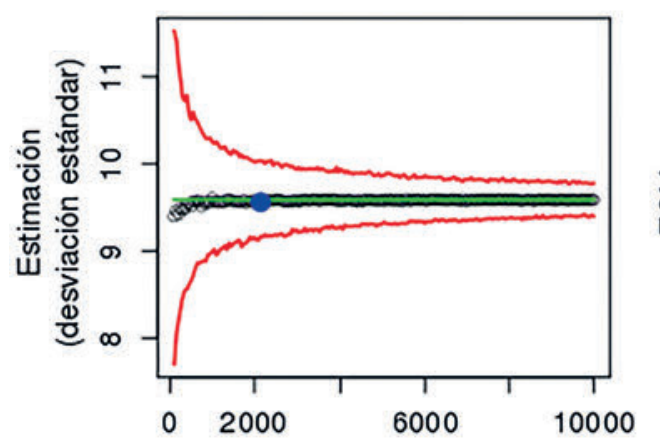

tamaño de muestra

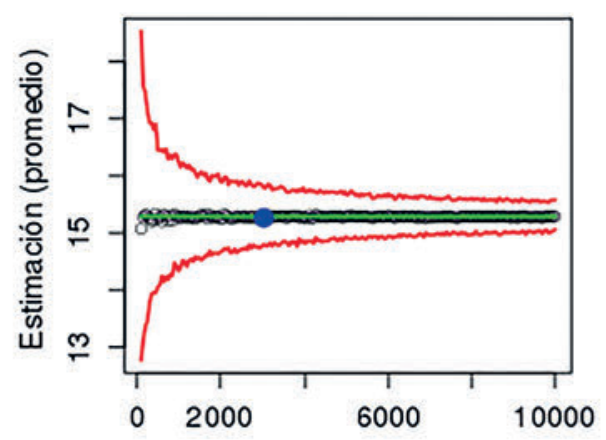

tamaño de muestra

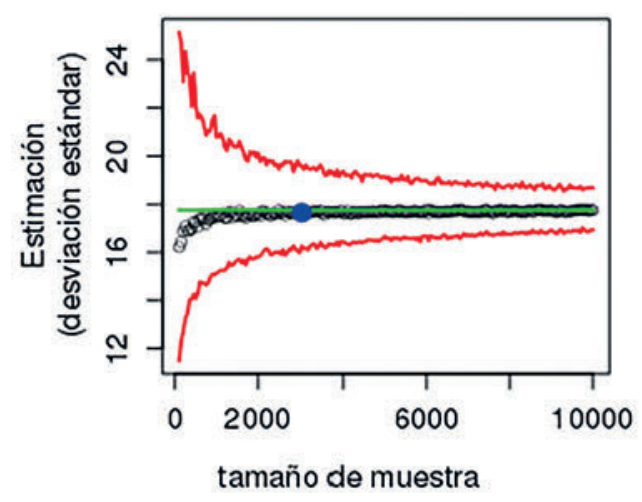

Figura 2.6b. Cálculos de bandas de confianza y error cuadrático medio (ECM) para el promedio y la desviación estándar de la duración del viaje a escala anual, para un ejemplo donde $\mathrm{N}=57000$. A la izquierda: estimaciones del promedio (arriba) y la desviación estándar (abajo) para cada tamaño de muestra. El valor verdadero está representado por una línea horizontal verde. Las bandas de confianza están en rojo. Los valores estimados se representan por circunferencias negras. A la derecha: error cuadrático medio para el promedio (arriba) y la desviación estándar (abajo), para cada tamaño de muestra. En todos los casos, el valor azul indica el valor óptimo para esa réplica.

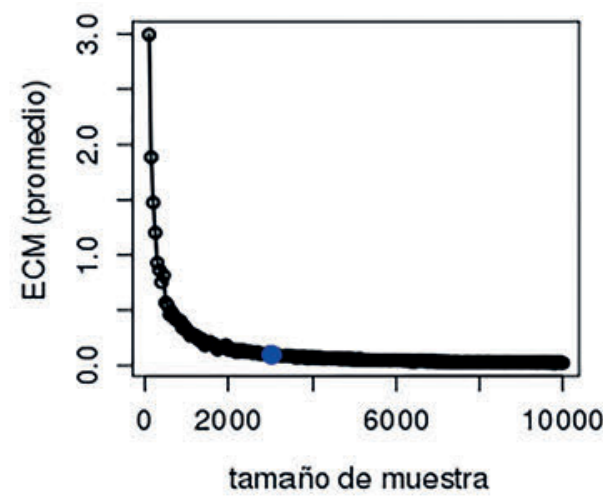

Figura 2.7. Cálculos de bandas de confianza y error cuadrático medio (ECM) para el promedio y la desviación estándar de la captura por unidad de esfuerzo, para un ejemplo donde $\mathrm{N}=57000$. A la izquierda: estimaciones del promedio (arriba) y la desviación estándar (abajo) para cada tamaño de muestra. El valor verdadero está representado por una línea horizontal verde. Las bandas de confianza están en rojo. Los valores estimados se representan por circunferencias negras. A la derecha: error cuadrático medio para el promedio (arriba) y la desviación estándar (abajo), para cada tamaño de muestra. En todos los casos, el valor azul indica el valor óptimo para esa réplica. 